\title{
Gradhiva
}

GRADHIV

Revue d'anthropologie et d'histoire des arts

\section{Ramasser le monde. Ce que les œuvres de la nature et de la culture font aux collectionneurs contemporains}

Collecting the world. What works of nature and culture do to contemporary collectors

Brigitte Derlon et Monique Jeudy-Ballini

\section{OpenEdition \\ Journals}

Édition électronique

URL : http://journals.openedition.org/gradhiva/3177

DOI : 10.4000/gradhiva.3177

ISSN : $1760-849 X$

Éditeur

Musée du quai Branly Jacques Chirac

Édition imprimée

Date de publication : 25 mai 2016

Pagination : 168-193

ISBN : 978-2-35744-093-7

ISSN : 0764-8928

\section{Référence électronique}

Brigitte Derlon et Monique Jeudy-Ballini, « Ramasser le monde. Ce que les œuvres de la nature et de la culture font aux collectionneurs contemporains », Gradhiva [En ligne], 23 | 2016, mis en ligne le 25 mai 2019, consulté le 30 avril 2019. URL : http://journals.openedition.org/gradhiva/3177 ; DOI : 10.4000/ gradhiva.3177 


\section{Ramasser le monde}

Ce que les œuvres de la nature et de la culture font aux collectionneurs contemporains

par Brigitte Derlon et Monique Jeudy-Ballini

Collectionner à la fois l'art primitif et les spécimens naturels engage à ces deux types d'objets, des relations moins dissemblables qu'on pourrait le supposer. De fait, l'approche ethnographique rend sensible aux continuités discursives, physiques et conceptuelles que les collectionneurs établissent entre les éléments de ces catégories au-delà de leur disparité. Représentations de la collecte, préférences esthétiques, disposition spatiale des pièces, analogisme, projections fusionnelles ou encore réflexions sur le fragment, la sélection et le temps: ces différents aspects, ici mis en perspective, relèvent d'un même regard intégrateur. Ils dessinent un imaginaire tout à la fois intime et cosmologique qui participe de la construction d'un rapport subjectif et donc forcément singulier au monde. 


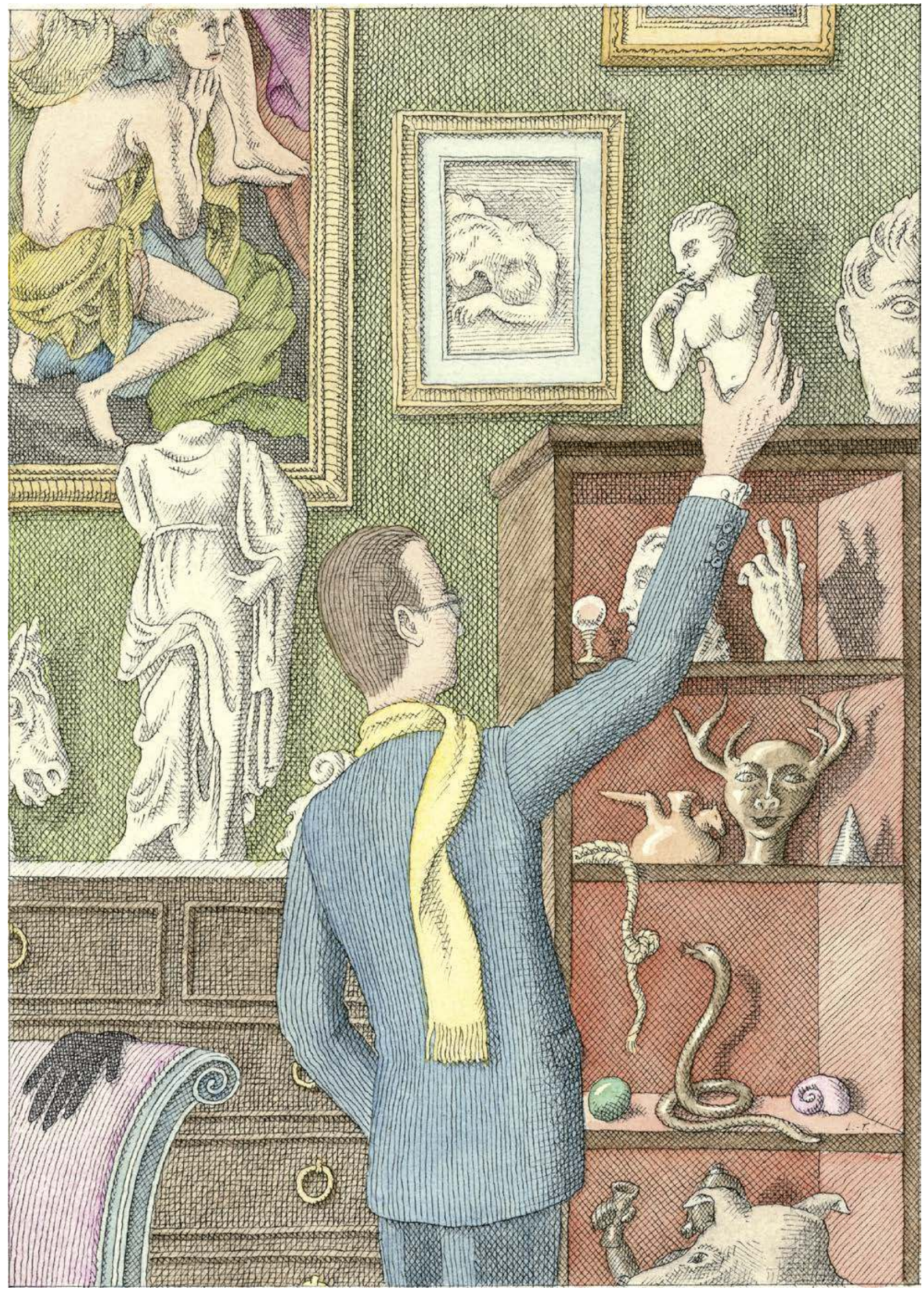


Les travaux sur les pratiques contemporaines de collection procèdent le plus souvent par approches généralistes ou thématiques, par catégories particulières d'objets ou encore focalisation sur un collectionneur célèbre. À moins d'être considérés comme des «collectionneurs de collections" (Cueco 1995) dont l'éclectisme même interpelle, ceux qui possèdent des ensembles d'objets disparates sont rarement interrogés sur le sens donné à ce choix, spécialement quand les pièces rassemblées relèvent à la fois de la nature et de la culture. Aussi a-t-on tendance à traiter séparément le collectionneur de reliquaires kota et le collectionneur de fossiles, quand bien même il s'agit de la même personne. C'est au contraire sur ce qui rapproche et relie les diverses classes d'objets collectés aux yeux de leur détenteur que nous voudrions porter l'attention en tirant parti d'informations recueillies lors d'une précédente enquête sur les collectionneurs d'art primitif (Derlon et Jeudy-Ballini 2008).

Parmi ces derniers, certains ont développé parallèlement un intérêt pour ce que l'on appelait autrefois des naturalia: pierres, insectes, graines, coquillages... Ils partagent en cela les goûts de personnalités tels André Breton et Jacques Kerchache. Dans l'atelier de Breton, les artefacts océaniens ou amérindiens voisinaient avec des papillons, des coléoptères, des bois flottés, des racines, des agates, une carapace de pangolin, un oursin fossilisé et des os de seiche, entre autres. Moins éclectique et surtout connu pour sa passion de l'art primitif, Kerchache s'était aussi constitué des collections minéralogique, conchyliologique et entomologique.

On ne collectionne pas des appuie-nuques océaniens comme on collectionne des papillons. Les prix, le goût et les critères d'appréciation de ces objets, les connaissances qui leur sont associées mais aussi les circuits d'approvisionnement et les réseaux de sociabilité impliqués diffèrent fortement. Nous voudrions montrer cependant que cette disparité n'engage pas un rapport si différent que cela aux objets ni ne donne lieu à la constitution d'univers de collection étanches.

\section{Manières de dire}

Si nos interlocuteurs tiennent les diverses catégories d'objets accumulés pour autant de collections distinctes, ils laissent aussi implicitement entendre des formes de continuité entre éléments naturels et productions culturelles. Les métaphores relatives au mode d'acquisition des pièces d'art primitif sont particulièrement significatives de ce point de vue. Ainsi, la trouvaille d'un artefact est souvent dite en des termes évoquant le ramassage sur une plage ou la cueillette dans un champ. Un collectionneur compare sa quête d'objets anciens dans des villages africains à l'action de «chercher quelque chose sur le rivage». D'une cuillère achetée dans un village mais provenant d'une autre région, il affirme qu'elle est «comme échouée là par une marée ». À l'en croire, son désir de découverte trouve constamment de quoi se satisfaire puisque, dit-il, «il y a toujours des choses qui sortent, ça tient de la récolte». Un de ses confrères qui rapportait des antiquités de ses voyages explique, lui, qu'il les avait "glanées à l'étranger », employant un verbe réservé aux produits agricoles quand il concerne des choses. Un autre encore affirme «marcher les yeux baissés, muni d'un grand sac et d'une machette ", à la manière d'un chasseur ou d'un collecteur de spécimens naturels dans la brousse.

\footnotetext{
ci-contre

fig. 1

Pierre Le-Tan, Quelques

collectionneurs, 2013

(C) Pierre Le-Tan.
} 


\section{ci-contre}

fig. 2

Claude Parent-Saura Totem chamane, 2000 , bois peint, sac, tissu

kuba ancien, hauteur:

1, 86m. Photographie:

Steve Drevet et Claude

Parent-Saura
L'agencement des pièces d'art primitif dans l'espace domestique suscite des métaphores comparables. "C'est quasiment de la taxidermie, déclare un amateur. On sort quelque chose de son milieu naturel et on essaie de le faire revivre dans la collection. " La naturalisation, pratique consistant à donner l'illusion d'une apparence vivante à des animaux morts, sert à penser la volonté de doter l'objet d'un effet de présence au sein de la collection. En creux, se révèle une conception assimilant la collecte à une captation de vie et l'entrée en collection à une renaissance. Pour certains, on le soulignait ailleurs (ibid.), l'objet extrait de son site de provenance ne donne sa véritable mesure qu'abstrait de ses références originelles. «À mon sens, un objet ne peut vraiment revivre que dans un contexte réinventé", disait une collectionneuse.

De manière générale, on relève chez nos interlocuteurs une nette tendance à rapporter une sculpture à sa matérialité naturelle de "bout de bois", de «tronc à peine dégrossi » ou de «pierre à peine sculptée». Dans un mouvement symétrique et inverse, quelques-uns parlent d'un coquillage ou d'une pierre comme d'une «production» ou du résultat d'un «travail», à l'instar d'une réalisation humaine. Cette idée d'une nature industrieuse procédant d'une créativité comparable à celle des artistes se retrouve sous la plume de Claude Lévi-Strauss - dont on rappellera qu'il collectionnait l'art primitif:

J'aime errer sur la grève délaissée par la marée [...] en ramassant des cailloux percés, des coquillages dont l'usure a réformé la géométrie, ou des racines de roseaux figurant des chimères, et me faire un musée de tous ces débris: pour un bref instant il ne cède en rien à ceux où l'on a assemblé des chefs-d'œuvre; ces derniers proviennent d'ailleurs d'un travail qui - pour avoir son siège dans l'esprit et non au-dehors n'est peut-être pas fondamentalement différent de celui à quoi la nature se complaît. (Cité in Le Fur 1990: 92)

Dans les façons de parler de nos interlocuteurs, l'absence de coupure tranchée entre ce qui relèverait d'un ordre naturel et d'un ordre culturel peut aussi s'exprimer à travers certaines descriptions d'artefacts où les deux apparaissent indissociables:

J'ai beaucoup d'objets atypiques qui sont des questionnements. Ça, c'est une racine, un objet de Nouvelle-Guinée. C'est une racine qui avait une fonction un peu fétiche, sur laquelle il y a quantité d'inscriptions, de figures animales par endroits symboliques. Là, un petit zigzag; là, un arc avec des flèches. Un côté anthropomorphe et en même temps un côté serpent. Comment dire? C'est une petite séquence d'écriture, un peu comme un objet préhistorique. C'est une phrase... (Entretien, septembre 2004)

À propos d'une autre de ses pièces, le même collectionneur dira: «Regardez! Ça, ça vient d'Afrique. C'est une racine, une racine sur laquelle on a fixé de la ficelle et des bouts de tissu pour faire de la magie. " Alors même qu'on sait combien les amateurs d'art primitif se plaisent à mettre plutôt en avant les dimensions émotionnelles et spirituelles qui donnent leur plus-value aux objets, sa présentation triviale de la pièce insiste sur 


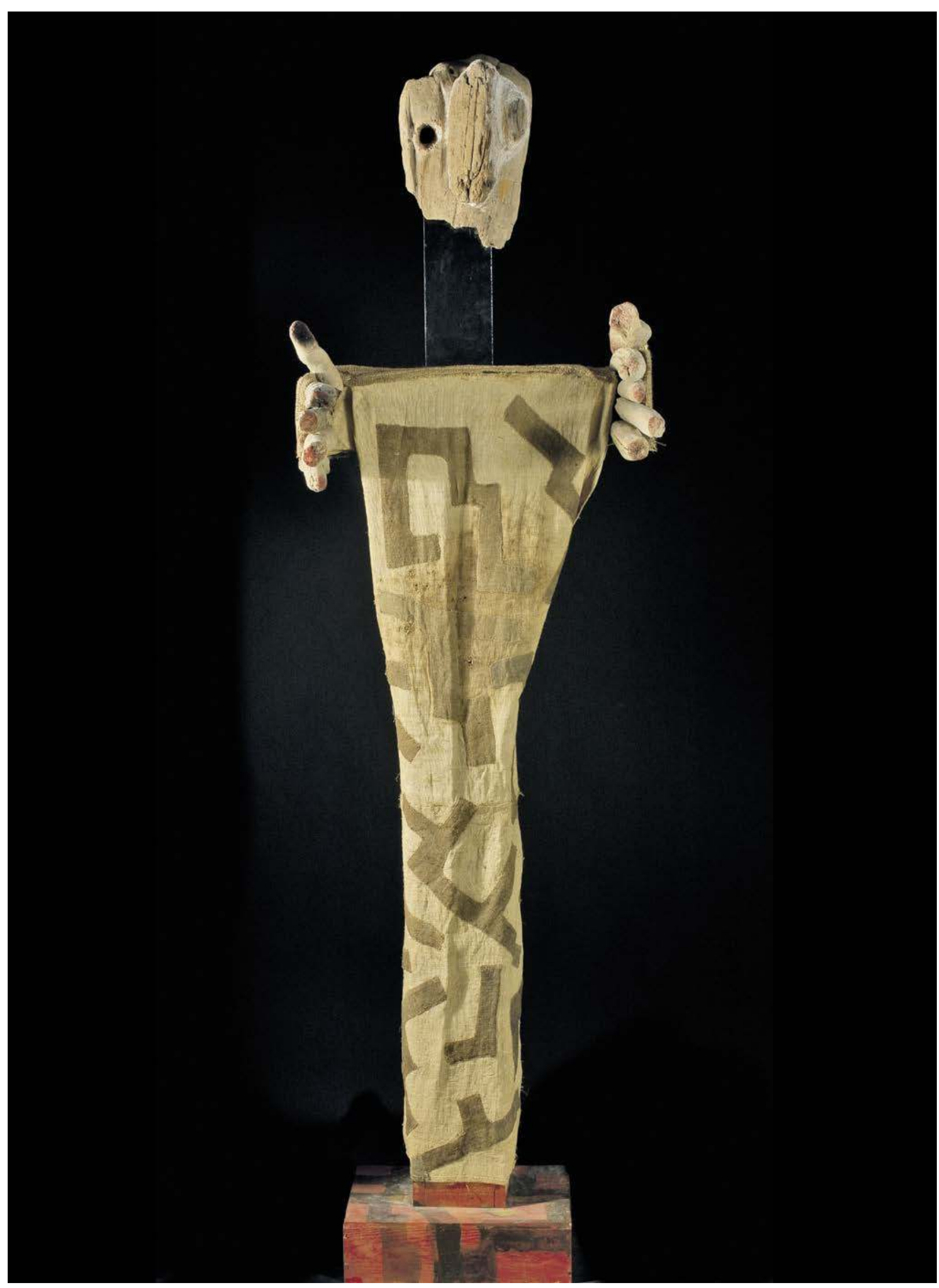


la racine qui en est le support brut. En projetant sur les autres ses propres conceptions, il déclare encore: «Les Africains ont l'humilité de nous rappeler que c'est d'abord un bout de bois qu'ils ont sculpté, une matière vivante. Ça ne les gêne pas de voir leurs sculptures se déliter et mourir. »

En comparant cette fois un objet naturel à une production culturelle, un autre collectionneur qui vécut longtemps en Afrique rapporte qu'un chef de village gabonais lui fit un jour cadeau d'une pierre, trouvée dans une rivière, qu'il avait précieusement conservée une trentaine d'années enveloppée dans un pagne au fond d'une cantine. «Les Africains gardent les branches en forme de crocodile, de serpent, on le sait. Mais les pierres, jamais! Et cette pierre est d'une telle modernité! Si on pouvait savoir pourquoi ce type a ramassé cette pierre... » Que cet objet singulier, «naturellement taillé comme un Brancusi », ait pu fasciner un villageois sans culture artistique européenne ne laisse pas de l'étonner.

\section{Manières de faire}

Ces rapprochements entre art et éléments naturels dépassent le seul registre discursif. Ils se trouvent aussi mis en œuvre physiquement, car il est rare que les différentes collections soient exposées sous forme d'ensembles séparés. La disposition des objets dans l'espace privé du collectionneur joue souvent sur des analogies: des analogies formelles, par exemple celle inspirant le choix de faire voisiner verticalement un rostre de poisson-scie et deux sculptures africaines longilignes, tous trois dotés de socles similaires; ou encore des analogies fonctionnelles, quand le rapprochement d'un vase à parfum antique, d'un réceptacle végétal à graines, d'une coquille de nautile, d'un œuf d'autruche et d'un crâne animal attire l'attention sur leur propriété commune de contenant. Un autre mode de mise en lien peut consister à créer une relation entre des collections différentes en les associant spatialement au même type d'objet. Dans un esprit proche des vanités, un collectionneur utilise ainsi des parties de squelettes d'animaux qu'il dispose, comme pour un jeu de piste, auprès de pièces choisies de ses collections d'objets naturels et d'art primitif: un bréchet d'oiseau planté dans une poterie sud-américaine emplie de sable, une carapace de crabe accrochée au bouton du tiroir d'une commode sur laquelle trônent des pièces africaines, un os long mêlé à des bois flottés...

Les cabinets de curiosités, ces ancêtres des musées où pouvaient cohabiter œuvres d'art, instruments scientifiques, minéraux et éléments de la faune ou de la flore, sont mobilisés à titre de référence par certains collectionneurs. S'ils s'en réclament parfois intellectuellement en affichant leur goût pour le merveilleux ou l'analogisme, ils s'en inspirent aussi souvent de façon très concrète dans le choix du mobilier et des dispositifs de présentation. L'un range sa collection minéralogique dans un de ces meubles à longs et fins tiroirs rappelant ceux des anciens cabinets, tandis qu'un autre a transformé un petit placard mural en «cabinet de curiosités du pauvre», selon ses termes, après avoir vu la célèbre peinture illusionniste de Domenico Remps (1690) dans L'Invention des musées de Roland Schaer. Immédiatement évocateur de son illustre modèle, le placard aux portes ouvertes expose crâne, vase, œuf, coquillage et réceptacle à graines dans autant de niches qui les mettent en valeur, ainsi que statuettes, végétaux séchés, 
pierres, dessins de papillons et petits objets en métal; soit, au dire de ce collectionneur, "des choses qui seules ont un intérêt limité mais qui prennent sens ensemble». Outre des affinités de fonction dont celle de contenant précédemment évoquée, les rapports de voisinage générés suggèrent des affinités de forme, de couleur, de matière et d'origine géographique. Artificialia, naturalia et exotica; durables ou fragiles; antiques ou récents; intacts ou fragmentaires; en pierre, métal, bois, terre, nacre ou papier; issus de différents continents: les objets choisis, dont chacun est rapporté à un souvenir ou à un événement biographique, composent un monde en miniature. Pour son détenteur, ce microcosme représente «un bilan de ce que peut offrir la nature tout simplement en la ramassant»- le mot «nature» incluant donc pour lui les créations humaines. À travers ses collectes et leur arrangement domestique, il «ramasse» le monde aux sens propre et figuré. Ses collections, il les voit comme «une boîte de Lego ouverte» qui susciterait des envies d'agencements et de mises en scène, comparables en cela aux briques de construction d'une cosmologie personnelle.

Si elle a perdu la finalité scientifique qui était la sienne à l'époque des cabinets de curiosités - «la similitude n'est plus la forme du savoir», assurait Michel Foucault (1966: 65) -, la quête analogique n'en conserve donc pas moins sa vitalité chez certains collectionneurs. Elle participe pour eux d'un mode de connaissance qui se veut distinct du rationalisme et relève d'un imaginaire à la subjectivité assumée. Elle se nourrit de leur passion pour la mise au jour de correspondances inédites entre les objets. Leur démarche n'est guère éloignée de celle de Breton, chez qui l'assemblage des pièces collectionnées (art primitif, art populaire, beaux-arts, objets naturels...) tenait d'un acte de création poétique cherchant à «relier les choses entre elles pour affirmer la continuité du monde», l'existence d'un «cosmos unifié » (Ottinger 2011).

Les coléoptères que Breton collectait en forêt, il les disposait dans des boîtes de naturaliste sans toutefois se conformer aux usages des entomologistes. Dans l'une d'entre elles, tapissée d'un fragment de carte routière et de feuilles de papier découpées, les insectes épinglés sur un mode artistique dessinent des formes colorées. Selon Bernard Dufour:

... son atelier, ses murs avaient le caractère de ses boîtes d'insectes, bourrés de haut en bas, de gauche à droite, organisés souvent par des étagères savamment agencées [...]. Aux yeux de Breton, [son atelier était] la boîte où il travaillait à son écriture, cette boîte qu'il remplissait sans cesse, y fabriquant des relations de voisinage qu'il laissait foisonner et créer du sens. (Dufour 1994: 21 et 23)

En somme, si l'approche analogique joue à l'échelle des rapports entre les pièces de la collection, elle joue aussi à l'échelle des liens entre la partie (boîte de coléoptères) et le tout (atelier).

Il est encore des cas où la créativité qui opère dans l'assemblage des objets de nature et de culture trouve un prolongement dans la production d'œuvres d'art. Ainsi des réalisations plastiques de l'artiste et collectionneur d'antiquités amérindiennes Claude Parent-Saura, qui conjuguent des bois 


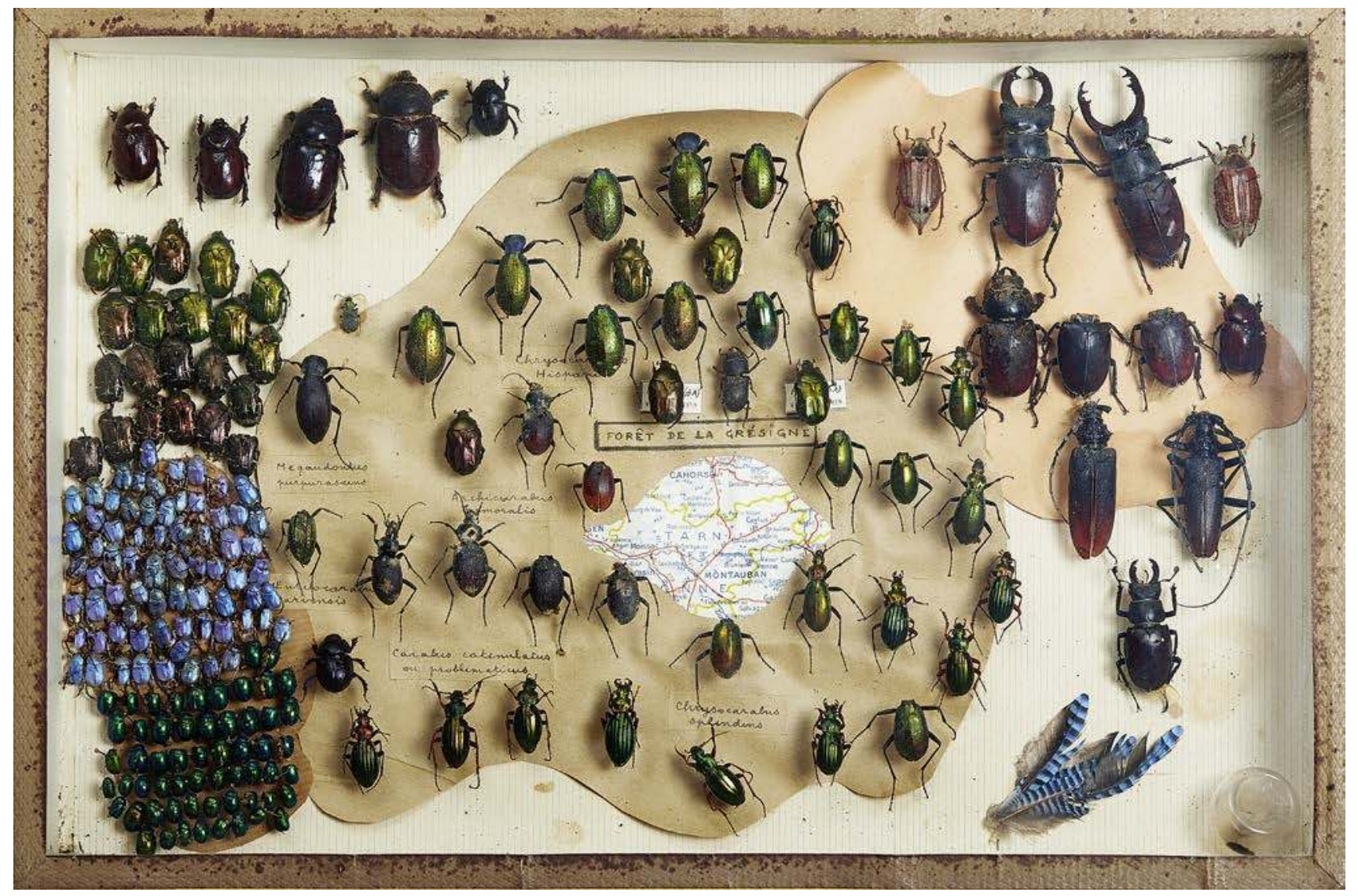


flottés, des morceaux de verre dépoli ou des coquillages ramassés sur la plage avec des fragments de tissu chancay ou de poterie anasazi. Les rapprochements ainsi induits entre formes et couleurs ont ovocation à célébrer l'esprit d'accord, la continuité, les «analogismes », dirait Parent-Saura (Costa [éd.] 2010).

\section{Le regard intégrateur}

Les continuités entre artefacts et objets naturels passent aussi par des correspondances imaginaires comme celles générées par la paréidolie, ce mécanisme cognitif jouant sur des illusions d'optique et consistant à identifier des formes dans des images aléatoires. Dans le domaine de la minéralogie, c'est lui qui rend compte de la fascination pour les pierres graphiques, ces variétés de calcaire ou de marbre porteuses de traces de leur genèse géologique propices à l'interprétation. Un collectionneur d'art primitif et de minéraux s'émerveillait des stratus, des ruines architecturales, des déserts stellaires ou des écritures inconnues qu'il reconnaissait à la surface de ses «pierres de rêve» - selon leur désignation chinoise. II s'était aménagé un atelier de coupe de pierre pour en extraire des tranches révélant d'éventuels paysages ou dessins scripturaires. En effet, comme l'observe Yves Le Fur, ces tranches une fois polies se prêtent à des perceptions, articulées sur les conventions picturales, qui amènent à discerner ciel, terre, horizon, lumière et profondeur de champ; des perceptions qui peuvent assimiler la brillance du polissage de la pierre à celle du vernis de la peinture à l'huile, ou les stries de la matière à des marques de coups de pinceau (Le Fur 1990: 105).

Aux yeux de ce collectionneur, l'une de ses pierres évoquait Voyage au centre de la Terre de Jules Verne et la découverte, par Axel et Lidenbrock, d'une mer souterraine bordée d'un ossuaire et de champignons géants. Dans les imprégnations métalliques d'une autre, à laquelle il faisait «subir une certaine inclinaison pour le repérer sous la loupe», il devinait un message cryptique venu d'Alpha du Centaure. De «ces objets qui résonnent en nous », il parlait avec enthousiasme: «ll y a des gars qui rêvent avec la Loterie nationale, moi je rêve avec des pierres! ». II s'enchantait tout autant des figures anthropomorphes ou zoomorphes qu'il décelait dans ses bois flottés et comparait à des œuvres d'art. Cette identification était si puissante qu'elle l'amena à faire exécuter une reproduction en bronze d'un bois flotté rappelant une antique statuette érodée. Soclée, elle trônait sur une étagère à côté de pièces d'art primitif comme s'il s'agissait d'un spécimen de plus. On ne sait qui de la nature ou de l'art, ici, imitait l'autre.

La continuité peut aussi se mesurer à l'aune de la beauté construite par le regard posé sur les objets de collection. La configuration d'une gousse végétale, l'architecture interne d'un coquillage, la transparence irisée d'une aile de papillon ou la construction plastique d'un masque primitif: tous sont susceptibles d'induire une égale admiration. "À ce stade de perfection, il n'y a plus de hiérarchie", confie l'un de nos interlocuteurs. Pour qui sait encore regarder, déclare Lévi-Strauss, « un oiseau, un scarabée, un papillon invitent à la même contemplation fervente que nous réservons au Tintoret ou à Rembrandt » (cité in Eribon 2009: 241). Kerchache, qui traquait la rareté et le chef-d'œuvre dans la nature aussi bien que dans l'art primitif, disait quant à lui: «Je regarde un insecte comme l'œuvre d'un artiste

\section{ci-contre}

fig. 3

Boîte de coléoptères provenant de la collection d'André Breton, s.d. Collection particulière, photo Nelly Blaya/Musée de Cahors Henri-Martin. 


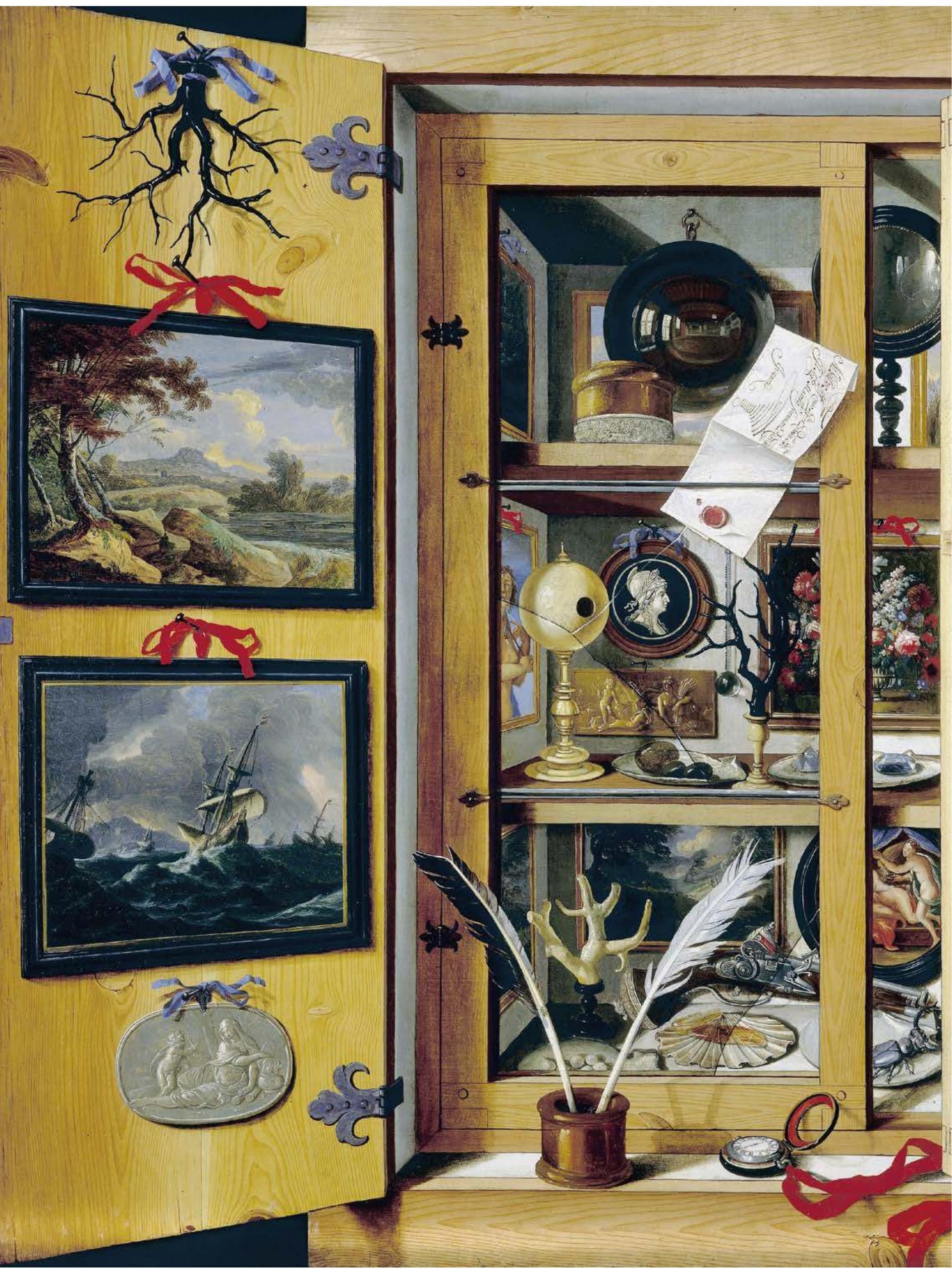




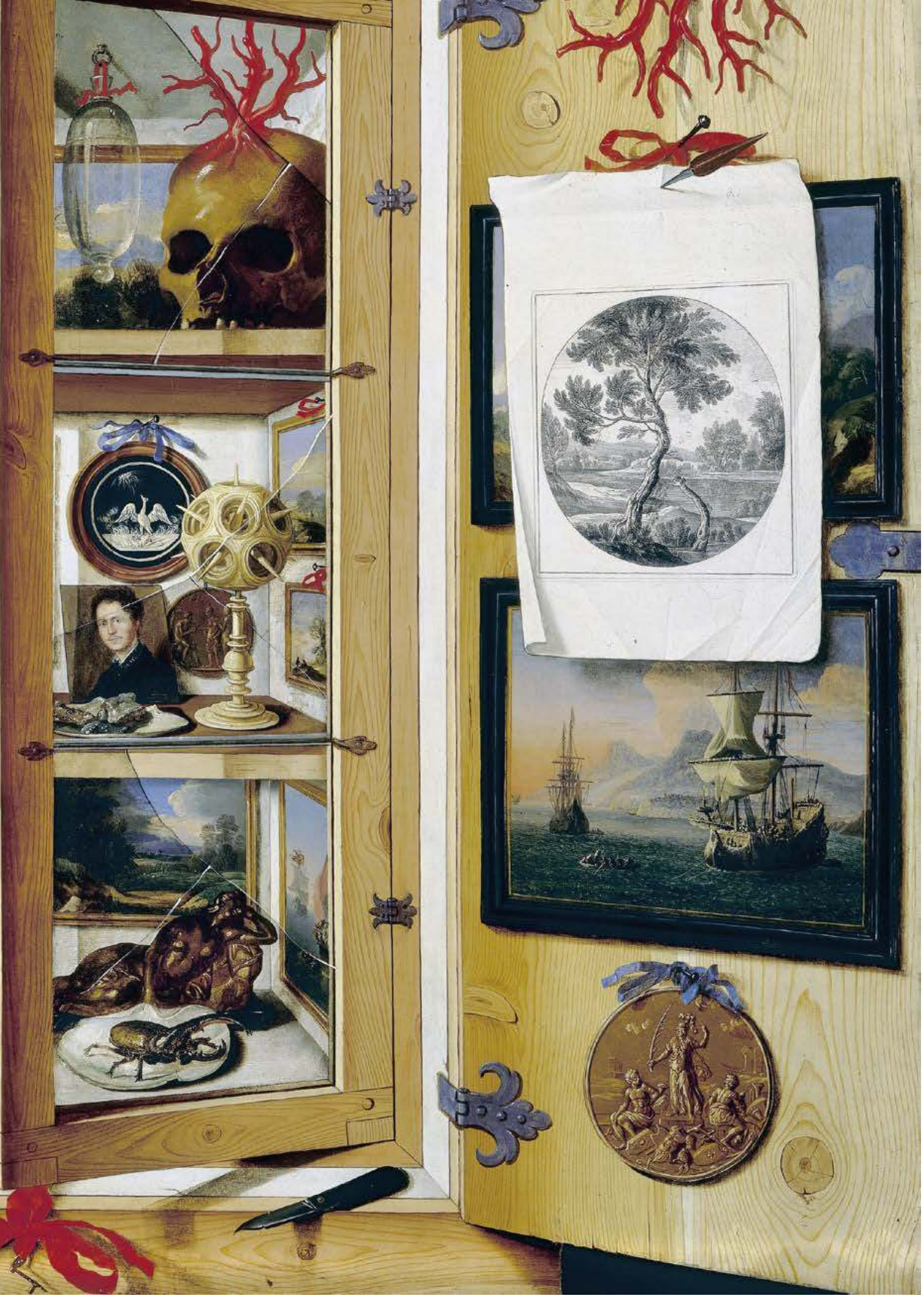

fig. 4

Domenico Remps, Cabinet de curiosité, vers 1690,

huile sur toile, $99 \times 137 \mathrm{~cm}$. Italie, Florence, Museo dell'Opificio delle Pietre Dure. Photo (c) Archives Alinari, Florence, Dist. RMN-Grand Palais / Georges Tatge. 
1. Caillois 2013 [1966] : $102 ; 1987[1970]: 121$.

2. Loisy, in Béthenod 2003b: 120

3. Ottinger 2011 . à part entière, porteur d'une identité qui lui est propre " (Kerchache Sottsass et Gries 2000). Ce sentiment l'amenait à se demander si l'on ne pouvait pas «embrasser d'un même regard l'ensemble des civilisations, des objets naturels et culturels " (Jean de Loisy, in Béthenod 2003b: 120). Sa collection entomologique débuta par l'achat à l'hôtel Drouot d'une centaine de boîtes dont il reclassa les insectes plusieurs années durant pour ne conserver que les «spécimens sublimes» (Anne Kerchache, in Béthenod 2003a: 30). II voyait chaque boîte "comme un tableau, comme une petite œuvre dont il serait l'auteur, et qu'il n'hésitait pas à refaire dix fois pour arriver à la perfection" (Loisy, in Béthenod 2003b: 122). Appropriée par le regard créateur, l'œuvre de la nature devient celle du collectionneur. De même que la boîte de coléoptères de Breton et la reproduction en bronze du bois flotté évoquées plus haut, une telle œuvre pourrait donner lieu à signature. Ce faisant, elle s'inscrirait dans la continuité des pierres de rêve les plus remarquables que les Chinois du xIx $x^{e}$ siècle sélectionnaient, encadraient, dotaient d'un titre et gravaient de leur cachet (Caillois 1987 [1970]: 47); ou encore des paésines florentines que les artistes utilisaient en guise de support à leurs peintures «pour profiter des décors que leur offraient libéralement les jeux de la nature» $(w: 33)$.

Collectionneur de minéraux, Roger Caillois tenait les productions naturelles pour la "référence ultime de toute beauté » (Caillois 1976 [1962] : 24). Selon lui, «l'univers des formes est un» et «la culture n'y dessine aucune extra-territorialité: elle n'en est qu'un cas particulier " (Jenny 1992: 60). En ramenant toutes les formes existantes à quatre grands types suivant les modalités de leur genèse (par accident, par croissance, par projet ou par moule), son «esthétique généralisée " fait fi de la distinction entre œuvres de nature et œuvres de culture.

"Même trame» ou "syntaxe générale» (Caillois ${ }^{1}$ ), «regard global» $\left(\right.$ Kerchache $\left.^{2}\right)$, «cosmos unifié» (Breton $\left.{ }^{3}\right)$ : le monde ne serait jamais qu'une totalité intégrée.

\section{Le temps et la mort}

La collection, dont l'arche de Noé pourrait tenir lieu d'emblème, a été interprétée comme un "unique bastion contre le déluge du temps" (Elsner et Cardinal 1994: 1) et une tentative pour transcender le caractère éphémère de la vie humaine. Former des séries, réunir, ordonner et désassembler des objets, les acquérir et s'en séparer créerait l'illusion de maîtriser le temps. Jean Baudrillard, qui défend cette conception, écrit:

Répertoriant le temps en termes fixes qu'elle peut faire jouer réversiblement, la collection figure le perpétuel recommencement d'un cycle dirigé, où l'homme se donne à chaque instant et à coup sûr, partant de n'importe quel terme et sûr d'y revenir, le jeu de la naissance et de la mort. [...] Ce que l'homme trouve dans les objets, ce n'est pas l'assurance de se survivre, c'est de vivre dès maintenant continuellement sur un mode cyclique et contrôlé le processus de son existence et de dépasser ainsi symboliquement cette existence réelle dont l'événement irréversible lui échappe. (Baudrillard 1968: 115 et 117) 
Chez le détenteur du cabinet de curiosités du pauvre évoqué plus haut, l'activité de collection participe, tout à fait sciemment, d'une réflexion sur le temps et la mort dans laquelle les objets naturels jouent un rôle primordial, et qui s'étend à l'ensemble de ses collections. L'une d'elles consiste en coquillages, non pas des espèces rares et recherchées mais de vulgaires bigorneaux ramassés sur une plage de Normandie. «J'aime faire jouer les formes, mais surtout les états ", dit-il. Sa manière de disposer ses trouvailles illustre les effets du temps en faisant de chaque spécimen la représentation d'un moment arrêté de leur inéluctable transformation:

Les coquillages me parlent du temps. Ce qui m'amuse, en constituant des séries, ce n'est pas de rassembler «ethniquement» des coquillages. Ce qui m'intéresse, c'est d'avoir l'image du temps. J'aime partir d'un coquillage beau et neuf en passant par un cassé pour arriver à des fragments tout usés. J'aime faire des carrés de coquillages de dix centimètres sur dix, de sorte que les objets se lisent des plus vieux, qui sont aussi les plus nacrés, aux plus jeunes, les moins abîmés. La collection d'insectes de Kerchache, elle en jette, mais c'est à la portée de tout le monde. Moi, j'essaie de tirer des conclusions de ma collection de bigorneaux: délimiter un autre temps que le nôtre, de la naissance au zénith jusqu'à la mort et à la réutilisation. (Entretien, avril 1999)

Le choix des objets et le principe gouvernant leur regroupement sont subordonnés à une conception cyclique du temps. Alors que les bigorneaux s'opposent aux insectes de Kerchache comme le trivial au précieux, les propos tenus laissent à penser que l'inverse s'appliquerait presque à leurs collections respectives. Le pur esthétisme de l'une la ravalerait dans le registre du banal tandis que la profondeur métaphysique de l'autre la hisserait vers le remarquable. Que notre interlocuteur valorise l'ordonnancement des bigorneaux en tant que construction intellectuelle ne l'empêche pas de le concevoir en même temps comme la transposition de phénomènes observables dans la nature:

Au bord de la mer, on découvre des cimetières de coquillages, de crabes. En plongée aussi, on découvre parfois des bouts de coquillages en suspension, des cadavres en décomposition, des poissons morts qui dansent, des oiseaux dont il ne reste que des plumes, prises dans le flux et le reflux. Ils flottent à mi-hauteur dans un nuage blanc au-dessus des algues. C'est très impressionnant, très beau, métaphysique. Ce sont des usines à transformations que l'on trouve dans la nature: du vivant au mort et du mort au vivant. C'est surprenant de tomber sur ces mises en scène naturelles. C'est ce que je cherche à faire dans mes propres mises en scène. L'art, c'est la volonté de refaire, c'est toujours aller dans le sens de la nature. (Ibid.)

Alors que ses séries de coquillages relèvent d'une expression de sa subjectivité, il entend s'effacer pour conférer à l'art, et à l'activité de collection qui en est partie prenante à ses yeux, le sens d'une re-production de la nature. Peut-être faudrait-il dire plus justement que, de son point de vue, ce qui le distingue à travers sa collection et rend celle-ci supérieure à toute 


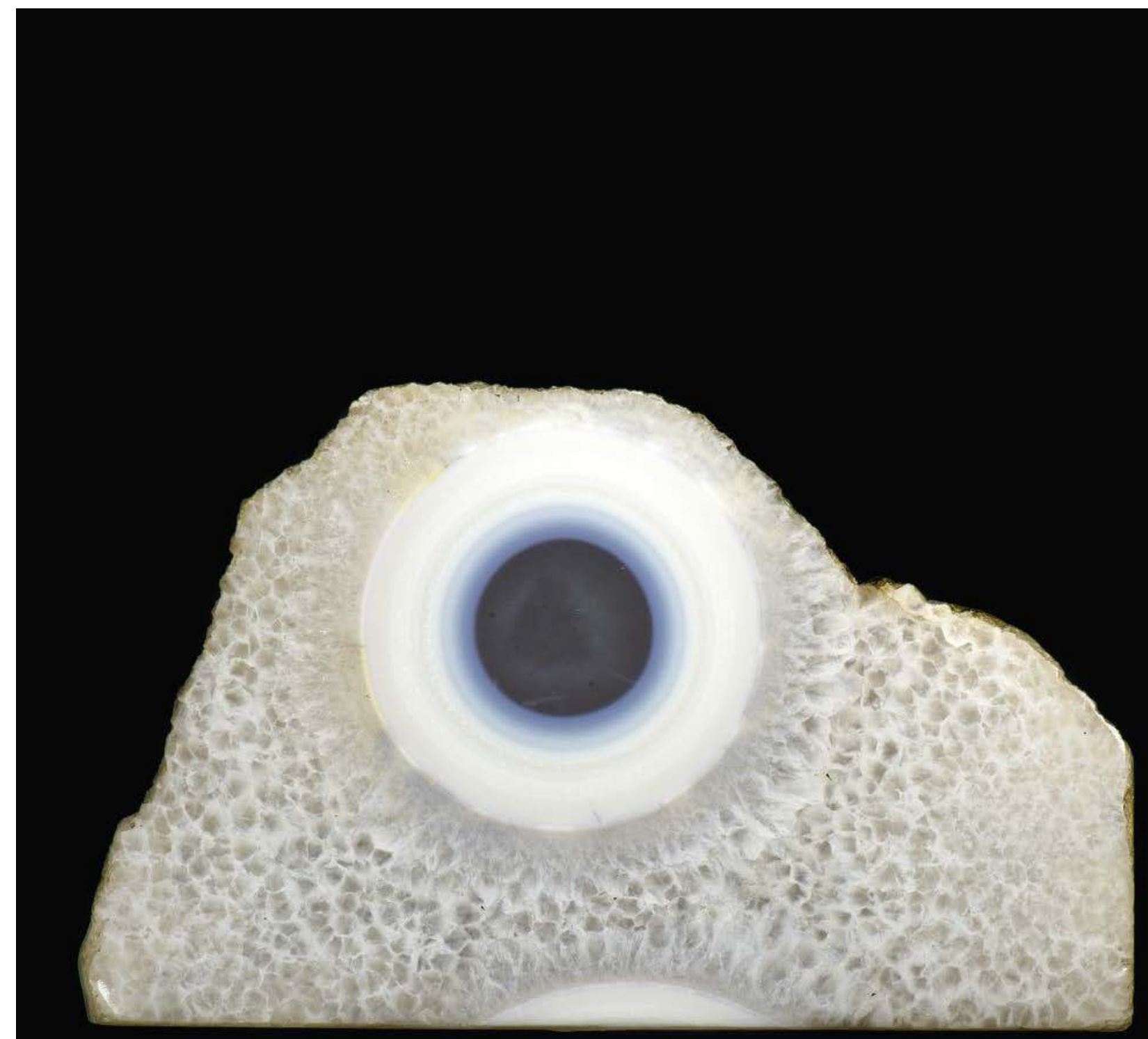


autre fondée sur l'esthétisme tient à sa pleine conscience de l'impossibilité pour l'art de s'affranchir de la nature. À l'instar de sa collection de coquillages incluant de simples bigorneaux, sa collection de pierres comporte des spécimens qu'il aime appeler des «cailloux» pour en souligner l'apparence ordinaire. «Dans les cailloux, assure-t-il, le phénomène du temps est plus difficile à voir. Il joue, mais sur une échelle plus longue, et c'est passionnant. » En montrant une pierre bicolore, il poursuit:

Ce sont deux roches qui se sont mélangées. C'est un bout de géologie. Et c'est ça qui m'intéresse! Pas sa beauté ou sa rareté. Quand j'étais aux États-Unis, je me suis rendu compte en me promenant dans le désert de Californie, en voyant les énormes rochers fendus, que c'était la même force géologique qui s'était exercée sur mon caillou; une force extrêmement lente, mais qui peut devenir rapide, comme dans le tremblement de terre de Los Angeles où j'étais à ce momentlà. Les murs ondulaient, c'était extraordinaire! C'est la même force qui tasse les cailloux. Mon caillou est un moment dans l'histoire géologique, un jour il deviendra du sable. Pour l'instant, il est arrêté. (Ibid.)

Rattaché en amont à la roche dont il provient et en aval aux fines particules auxquelles il sera un jour réduit, ce caillou qui conserve la marque des mouvements telluriques à l'origine de sa formation tient lieu, en somme, d'espace et de marqueur de temps. Son existence est rapportée à celle d'un point figé sur un continuum dont l'immobilité n'est qu'une illusion; une illusion due à la lenteur des transformations géologiques et au fait que sa présence au sein de la collection le protège de l'érosion en le maintenant dans une sorte de hors-temps. De manière significative, ce collectionneur qui se plaît à montrer les traces laissées par le passage du temps sur ses pièces d'art primitif (usure, partie fendue ou manquante, effacement du motif, réparation) dira plus tard regretter que ces mêmes objets, arrachés par la collecte à leur site d'origine, aient été ainsi soustraits à leur disparition «naturelle».

Chacune de ses collections évoque donc une échelle de temps et un cycle de transformations spécifiques: le temps «géologique» de la formation des roches, le temps long de la désagrégation en sable des cailloux et des coquillages, le temps moyen de la détérioration des artefacts créés par l'homme et, enfin, le temps court, biologique, du développement des plantes qui naissent et se reproduisent par bouturage au sein de son appartement. Collectionner des choses fragiles et vivantes comme les végétaux lui permet sans doute de s'accommoder du paradoxe d'une pratique consistant à rassembler des objets arrachés à leur processus naturel de transformation, mais intellectuellement construite autour de la valorisation de ce même processus: «La nature nous dit que les choses ne s'arrêtent jamais, je n'ai pas envie de la faire mentir par ma collection. »

\section{Sélection et jeux sériels}

Tandis que, jeune adulte, il ramassait des «pierres blanches sur une plage composée majoritairement de pierres grises», ce collectionneur eut l'impression d'« une sorte de ségrégation, apparemment sans raison, sinon un déterminisme qui fait choisir le blanc parce que c'est l'exceptionnel ». Il eut alors le sentiment dérangeant d'une forme de continuité possible entre

ci-contre

fig. 5

Agate de la collection

de Roger Caillois (C) MNHN/ F. Farges. 
4. Notons que c'est Georges Vacher de Lapouge qui aurait introduit le mot "ethnie» dans la langue française.

\section{ci-contre}

fig. 6

Paesine de la collection de Roger Caillois (C) MNHN/ F. Farges. sa pratique a priori anodine de collecte sélective et la hiérarchisation des humains en jeu dans le racisme et l'antisémitisme. De fait, on connaît l'importance que les sciences de la classification eurent dans la formation du racisme. Ainsi que le rappelle Yves Delaporte, "ce n'est pas un hasard si Vacher de Lapouge, connu surtout comme théoricien de l'aryanisme, a été l'un des plus éminents carabologues de l'histoire de l'entomologie 4 " (Delaporte 1984: 1237). Cette perspective parut si intolérable à notre interlocuteur, qui se défendait de rassembler «ethniquement » des coquillages en les organisant en séries, qu'il se mit soudain à ramasser aussi puis surtout des cailloux gris:

Car ce qui semble valide pour les pierres, la sélection, est inacceptable pour les hommes. En ce moment, je lis un livre sur le procès d'Eichmann et... la manière de choisir les juifs, les bons, les pas bons... Choisir un caillou c'est encore innocent mais le système, lui, ne l'est pas. (Ibid.)

Sa difficulté à assumer l'idée de choix par refus de l'exclusion, difficulté guère attendue de la part d'un collectionneur, explique la manière singulière dont il s'est procuré par la suite ses pièces d'antiquités et d'art primitif:

Je reprends les fins de collection, les fins de série. J'achète les objets dont mes copains ne veulent plus: un masque bambara pas terrible; une sculpture dogon abîmée - elle a une tête inhabituelle; un tambouralligator pas très beau; un vase étrusque recollé. (Ibid.)

En somme, qu'il collecte des coquillages ou achète des œuvres d'art, il choisit toujours ce que les autres éliminent: le banal, l'imparfait. À la fin d'un long entretien, cette préoccupation quant à la sélection s'éclaira quand il signala de manière anodine qu'il avait été un enfant (rejeté puis) adopté. Autrement dit, la continuité qu'il cultive entre objets de nature et objets de culture, il la ressent aussi entre objets de collection et êtres humains.

Chez quelques collectionneurs, une minorité dans notre enquête, le choix des pièces est dicté par l'engouement pour les séries et les jeux de variations sur un même thème. L'un d'entre eux collectionne notamment graines, cailloux, biberons, cartes postales de la forêt de Fontainebleau, tickets de métro, étuis péniens d'Océanie, masques inuits. II dit aussi faire collection "dans sa tête» des numéros de plaques minéralogiques qu'il ne peut s'empêcher de mémoriser quand il se trouve dans la rue. Son obsession pour les séries s'applique à tout, assure-t-il, sauf aux timbres... Elle l'a conduit à amasser près de dix mille boîtes de médicaments et s'exprime à travers la recherche passionnée de la plus petite variation. Pour lui, la motivation sérielle en vient à abolir la perception des différences de nature entre les objets et rend compte de l'égal transport affectif que tous lui inspirent.

\section{Mystique de la matière}

Plusieurs aspects du rapport à l'objet mis en évidence à propos des collectionneurs d'art primitif (Derlon et Jeudy-Ballini 2008), en particulier leur attrait pour l'inconnu et leur identification aux pièces collectionnées, trouvent des résonances flagrantes avec ce qu'on sait des amateurs d'objets naturels. 


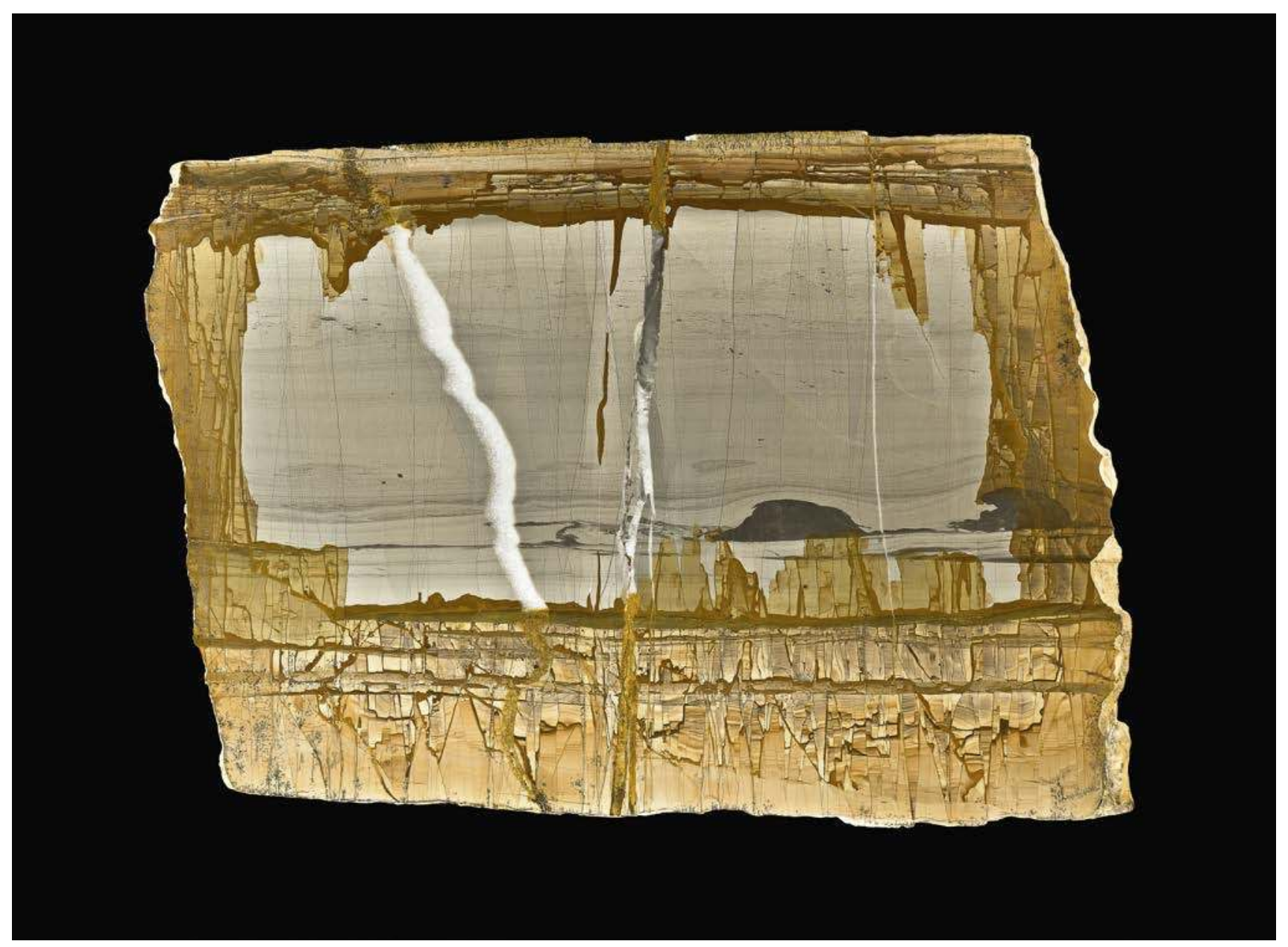




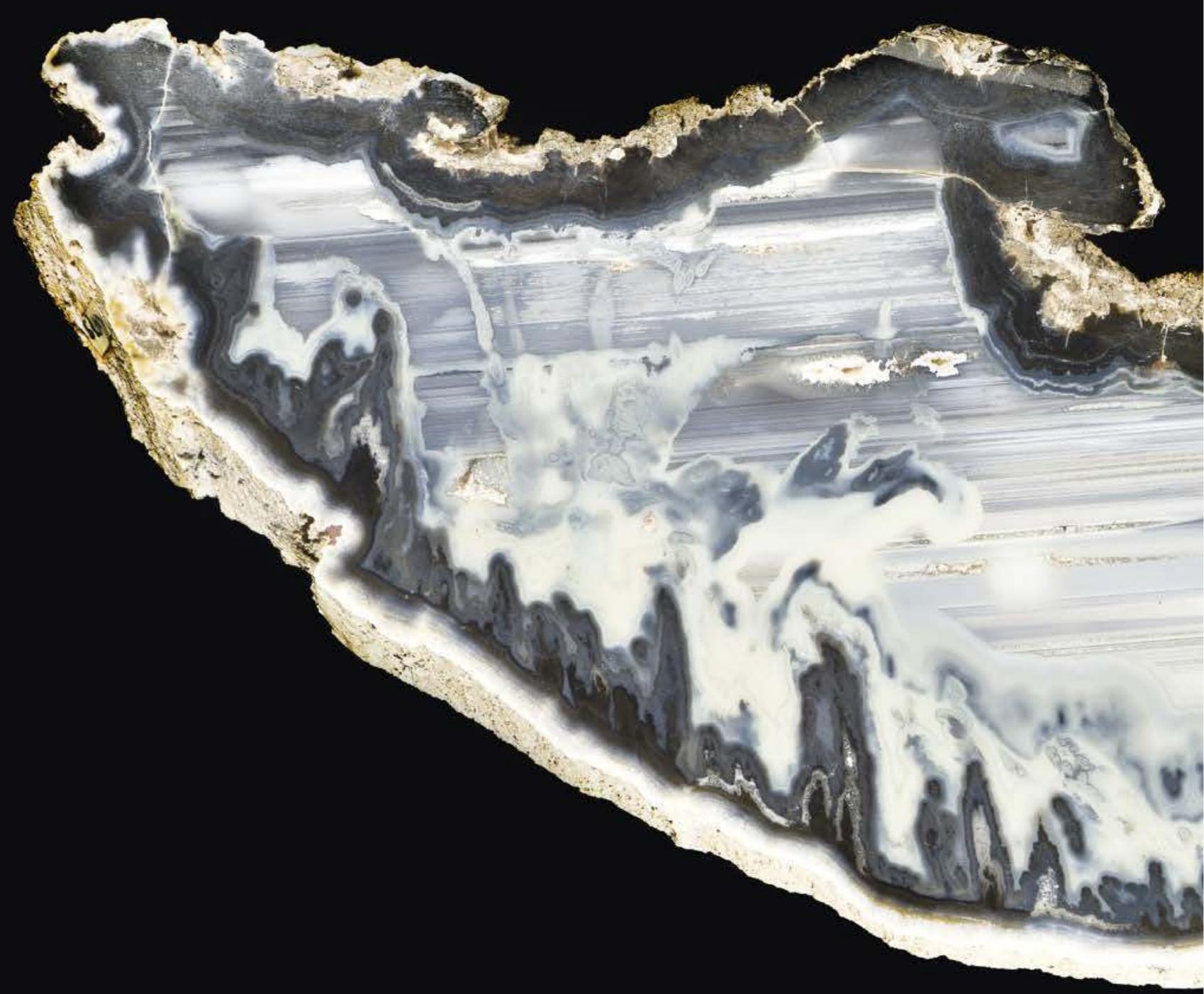




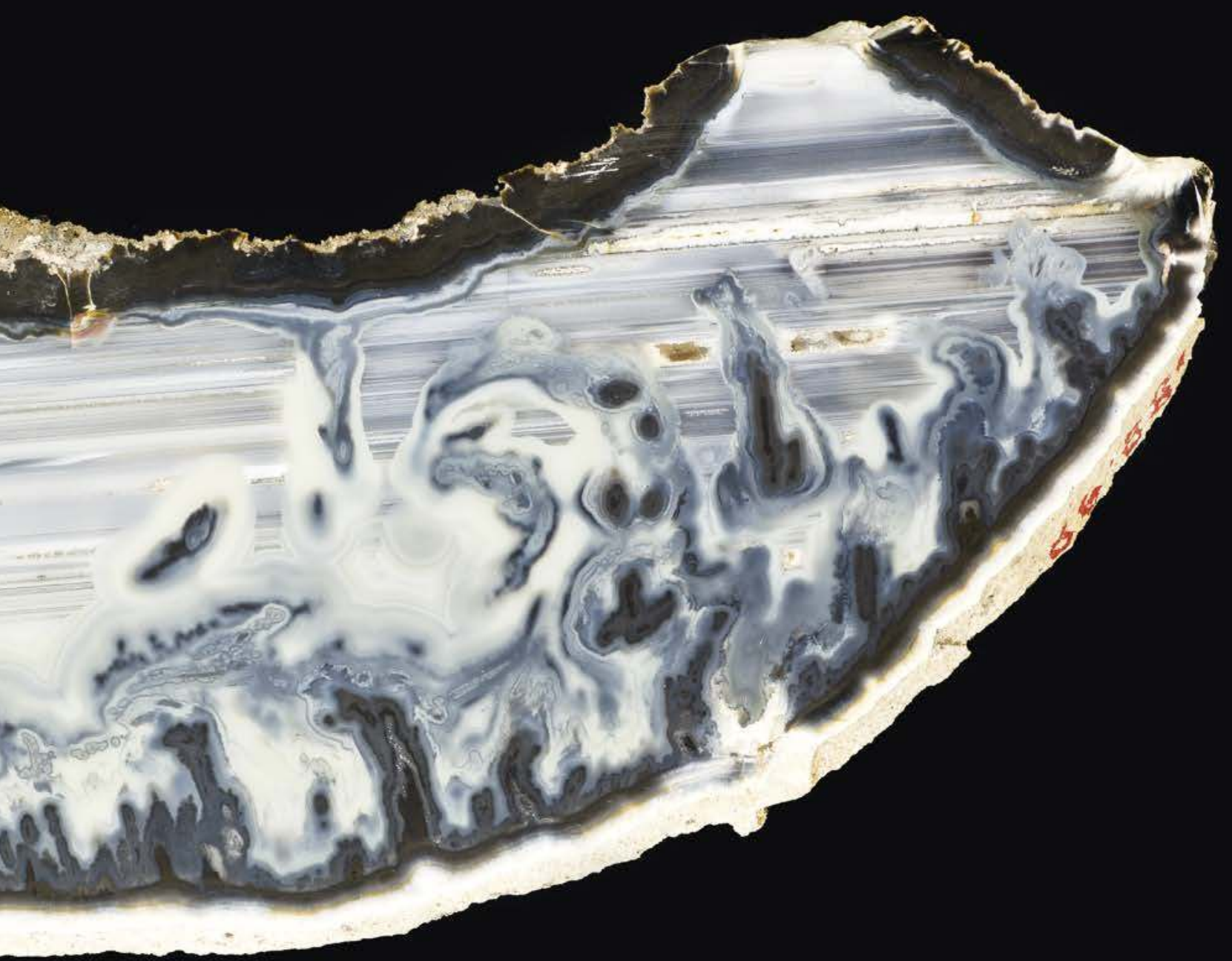


double page précédente

fig. 7

Agate dite "Le Navire»,

collection de Roger Caillois

(C) MNHN/F. Farges.
La fascination exercée par l'art primitif tient souvent à son aura d'étrangeté, nombre de ses collectionneurs se plaisant à penser qu'il les met en prise avec un ailleurs géographique, temporel et mental. Tout ce qu'ils ignorent à son propos (âge, lieu d'origine, signification, usage, identité de l'artiste, conditions de sa collecte...) en fait une porte ouverte sur le mystère et stimule les projections imaginaires. Ceux d'entre eux qui possèdent aussi des collections naturelles les investissent parfois de la même manière en exprimant leur sentiment d'être confrontés à des fragments énigmatiques. "J'ai une dilection pour les débris, les morceaux", affirme un amateur de pierres, de bois flottés et de sculptures incomplètes. Son esprit s'enflamme à l'évocation du rocher, de l'arbre ou de l'effigie à jamais inconnaissable dont chacune de ses possessions s'est un jour détachée. «J'aime bien les bouts de machins mystérieux, cette pierre avec sa forme spécifique et puis cette poterie aztèque", remarque l'un de ses confrères. Pour lui, pierre et poterie relèvent pareillement du fragment: la pierre, parce qu'elle s'est désolidarisée d'une masse rocheuse; la poterie, parce qu'elle est le reste d'une culture matérielle disparue et que, même intact, un ustensile s'inscrit toujours dans un système d'objets. Pierre et poterie apparaissent encore mystérieuses car ni l'histoire de leur production ni celle de leurs déplacements jusqu'à ce jour ne lui sont connues.

Dans la manière la plus fantasmatique de la vivre, la relation du collectionneur d'art primitif aux objets de sa passion se dit comme un brouillage des frontières entre personne et artefact. L'objet devient un sujet agissant. Doté d'intentionnalité, il choisit son détenteur et le place en position de passivité. C'est lui qui se signale à son attention, l'interpelle, lui «saute à la gueule ", "fait un clin d'œil ». "Les objets viennent à moi », disait George Ortiz. À ces mots font puissamment écho les écrits de Breton relatifs à ses découvertes d'admirables agates le long du Lot. Quand plusieurs, «d'une beauté inespérée", lui "sautèrent aux yeux", il imagina qu'«à chaque pas de toujours plus belles allaient s'offrir » et éprouva «la parfaite illusion de fouler le sol du paradis terrestre" (Breton 1970: 151). II célèbre ailleurs le rapport quasiment sexuel l'unissant à l'un de ces minéraux:

L'agate est venue me prendre à deux heures. Elle paraissait nerveuse et ses veines roses et bleues ne me donnaient d'autres pensées que celles que j'ai toujours. L'agate s'étendit nonchalamment sur le divan jonché de primevères; elle me regarda longtemps. J'étais nu jusqu'à la ceinture [...]. (Cité in Béhar 2014: 82)

La relation du collectionneur à ses pièces d'art primitif est marquée par un processus identificatoire faisant qu'à l'anthropomorphisation de l'un répond la chosification de l'autre. Un désir de fusion opère, qui s'exprime parfois par la sensation d'englober l'objet ou d'être englobé par lui. C'est là un phénomène également relevé dans la littérature relative aux amateurs d'insectes ou de minéraux. Quand elle cherche des spécimens dans la nature, la collectionneuse et artiste illustratrice Cornelia Hesse-Honegger a l'impression «de se perdre dans l'animal [...], de ressentir un lien profond, comme si, peut-être, elle-même avait été l'une de ces créatures - une punaise - et que son corps s'en souvenait» (Raffles 2010: 15, notre traduction). Une collectionneuse de papillons évoque quant à elle le sentiment d'être des leurs en 
contemplant dans un miroir l'image de sa peau «si pâle, presque bleutée, transparente [...], persuadée qu'elle appartient au monde insecte et qu'elle n'est encore qu'au stade de chenille. Elle attend avec impatience le jour où elle sortira de son cocon, étendra ses ailes colorées et s'envolera retrouver ses semblables» (Waldmann-Tozo 2005: 22).

Les minéraux, a priori moins propices aux projections fusionnelles, s'y prêtent pourtant aussi. Un texte chinois du début du $x{ }^{\mathrm{e}}{ }^{\mathrm{e}}$ siècle fait dire au détenteur âgé d'une pierre à images: «Moi, vieux, je suis la pierre et la pierre c'est moi» (Caillois 2013 [1966]: 77). Quand je les regarde attentivement, écrit Caillois en évoquant son propre rapport aux pièces de sa collection, «je me sens devenir de la nature des pierres. En même temps, je les rapproche de la mienne grâce aux propriétés insoupçonnées qu'il m'arrive de leur attribuer » (ibid. : 91). Pour lui, l'expérience mystique de la réaction «entre la fixité de la pierre et l'effervescence mentale» est de celles qui, certes, amènent l'âme «à se dissoudre dans quelque immensité inhumaine », mais dans un «abîme qui n'aurait rien de divin et serait même tout matière et matière seule» (ibid.).

\section{Subjectivités à l'œuvre}

En dépit de leur extrême diversité, les objets naturels et artificiels ne donnent pas lieu à des représentations ou traitements antithétiques. Frappent, au contraire, les similitudes du rapport à l'objet collectionné, qu'il relève du vivant ou de l'inerte, revête ou non une dimension culturelle, soit anthropomorphe ou pas, imposant ou minuscule, antique ou récent, unique ou commun, hors de prix, bon marché ou gratuit. Tous les amateurs ne conçoivent pas leurs collections sous l'angle d'un ensemble solidaire et signifiant à l'instar du créateur du cabinet de curiosités du pauvre. Mais un même esprit préside souvent au choix des pièces, qu'ils recherchent le chef-d'œuvre dans la sculpture primitive ou dans l'insecte, affectionnent le fragmentaire ou les rebuts, satisfassent un goût compulsif pour le sériel, mettent en scène une réflexion sur le rapport au temps ou voient encore dans leurs collections l'image en réduction d'un monde pensé comme une totalité intégrée.

On pourrait s'interroger sur l'existence d'une propension éventuelle à collectionner à la fois objets naturels et art primitif. Les possibles affinités formelles perçues entre eux, la fascination pour le mystère des origines, le goût pour l'étrange, ou le stéréotype consistant à rabattre les sociétés traditionnelles non occidentales du côté de la nature seraient autant de raisons de la présumer. II faut pourtant se garder des corrélations trop faciles. Les collectionneurs d'art primitif sont aussi, selon les cas rencontrés, des collectionneurs férus d'art contemporain, passionnés par les jouets anciens, les cartes postales ou les mécanismes d'horlogerie.

De fait, ce sont bien moins les propriétés objectives des choses qui rendent comptent de la composition des collections mixtes que le regard du collectionneur s'exerçant à retenir de ces choses une qualité commune qui, pour lui, fait valeur. La dimension sacrée souvent prisée dans l'art primitif, l'imaginaire peut aussi la rechercher - et par suite la détecter - dans des formes d'art auxquelles il n'est guère courant de l'associer. Ainsi du cubisme 


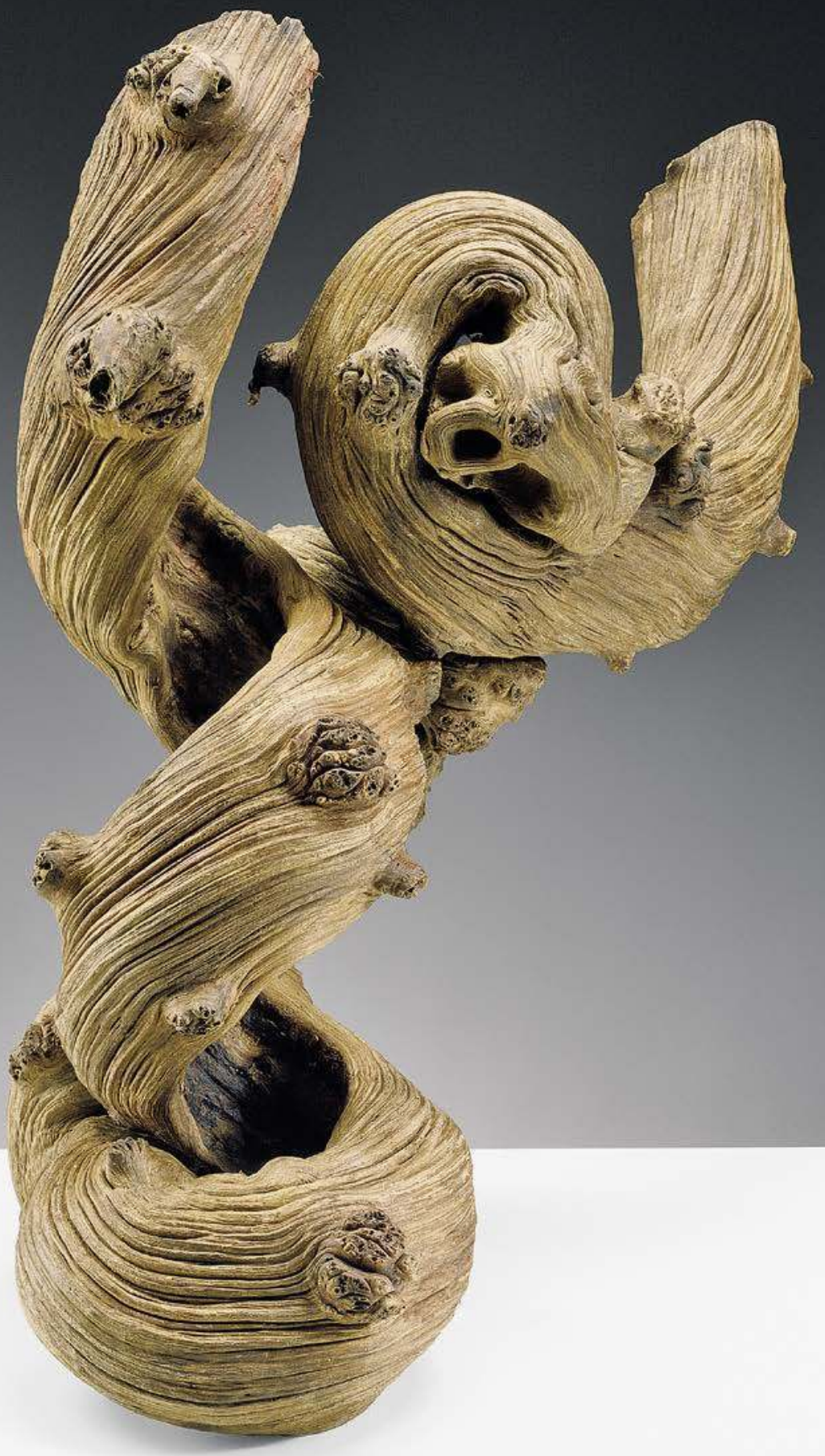


dans lequel Jean Paulhan voyait la «peinture sacrée» du $x x^{e}$ siècle (Paulhan 1993 [1956]: 11). Selon Raymond Court, «une peinture comme celle de Braque, en rendant la chose à la profondeur de son mystère et au fond dont elle surgit, rejoint la magie naturelle de l'objet sacré pour le primitif en retrouvant son opacité et son invisibilité ultime» (Court 2009: 634-635).

Le fameux adage de Marcel Duchamp voulant que «c'est le regardeur qui fait l'œuvre » dit assez que toute chose - donnée (objet naturel) ou créée (artefact) - est justiciable d'interprétations plurielles et qu'elle n'est jamais que ce qu'on en fait $\mathbf{5}$. De la même façon que tous les amateurs d'art primitif ne collectionnent pas les objets naturels et réciproquement, ceux qui collectionnent les deux, comme on l'a vu, ne le font pas nécessairement pour des considérations analogues. La vision qui les porte participe de la construction d'un rapport subjectif et donc forcément singulier au monde.

École des hautes études en sciences sociales / LAS derlon@ehess.fr

CNRS / LAS m.jeudy-ballini@college-de-france.fr
5. Un objet insignifiant ou une pièce de collection, un sujet de dégoût ou de délectation visuelle, par exemple. De nos jours il n'est jusqu'aux ordures ou aux excréments qui ne puissent se voir promus au rang de production artistique (Poubelles d'Arman, assemblages de détritus de Kurt

Schwitters, Merde d'artiste de Piero Manzoni).

Quant à la dimension esthétique, comme l'écrit Jean-Marie Schaeffer, elle "ne relève pas d'une propriété ontologique des objets mais d'un type d'activation mentale de ces objets " (Schaeffer 2004 : 35). 
Bibliographie

Ramasser le monde. Par Brigitte Derlon et Monique Jeudy-Ballini

\section{Baudrillard, Jean}

1968 Le Système des objets.

Paris, Gallimard.

\section{Béhar, Henri}

2014 La Maison de verre.

André Breton, initiateur découvreur. Paris, Éditions de l'Amateur-musée de Cahors Henri-Martin.

\section{Belk, Russell W.}

\section{1 [1995] Collecting in}

a Consumer Society. Londres et New York, Routledge.

\section{Béthenod, Martin}

2003a "Origines. Entretien avec Anne Kerchache", in Martin Béthenod (éd.), Jacques Kerchache. Portraits croisés. Paris, Gallimardmusée du quai Branly : 29-30.

2003b « Convoquer le merveilleux. Entretien avec Jean de Loisy, commissaire d'exposition ", in Martin Béthenod (éd.), Jacques Kerchache. Portraits croisés. Paris, Gallimard-musée du quai Branly: 119-122.

\section{Breton, André}

1970 Perspective cavalière.

Paris, Gallimard.

\section{Caillois, Roger}

1976 [1962] « Esthétique généralisée ", in Cohérences aventureuses. Paris,

Gallimard: 23, 68 .

1987 [1970] L'Écriture des pierres. Paris, Champs Flammarion.

\section{3 [1966] Pierres.}

Paris, Gallimard.

\section{Costa, Marie (éd.)}

2010 Les Analogismes de Claude Parent-Saura. Perpignan, direction de la culture de la Ville de Perpignan.

\section{Court, Raymond}

2009 "L'art et le sacré", Études 5(410): 627-638.

\section{Cueco, Henri \\ 1995 Le Collectionneur \\ Lévi-Strauss, Claude et Eribon, Didier}

de collections. Paris, Seuil.

\section{Delaporte, Yves}

1984 " Des insectes et des hommes", Les Temps modernes 450: 1235-1263.

\section{Derlon, Brigitte et} Jeudy-Ballini, Monique

2008 La Passion de l'art primitif. Enquête sur les collectionneurs. Paris, Gallimard.

\section{Dufour, Bernard}

1994 Des collectionneurs tels André Breton. Saint-Clémentde-Rivière, Fata Morgana.

\section{Elsner, John} et Cardinal, Roger

1994 «Introduction », in John Elsner et Roger Cardinal (éd.), The Cultures of Collecting. Londres, Reaktion

Books: 1-6.

\section{Foucault, Michel}

1966 Les Mots et les Choses. Paris, Gallimard.

\section{Jenny, Laurent}

1992 «Roger Caillois. Esthétique généralisée ou esthétique fantôme? ", Littérature 85: 59-73.

Kerchache, Jacques, Sottsass, Ettore et Gries, Patrick

2000 Nature démiurge. Insectes. Arles et Paris, Actes Sud-Fondation Cartier pour l'art contemporain.

\section{Le Fur, Yves}

1990 Résonances.

Paris, musée Dapper.
2009 De près et de loin. Paris, Odile Jacob.

\section{Ottinger, Didier}

2011 "Le mur de l'atelier " [en ligne], disponible sur: http://www.andrebreton.fr/ work/56600100228260\#noteN.

\section{Paulhan, Jean}

1993 [1956] Braque ou

la peinture sacrée. Paris, Éditions de l'Échoppe.

\section{Raffles, Hugh}

2010 Insectopedia. New York, Vintage: 15-40.

\section{Schaeffer, Jean-Marie}

2004 "Objets esthétiques? ?, L'Homme 170: 25-46.

\section{Waldmann-Tozo, Ella}

2005 "Forum ", Area Revue)s( 9 ("L'esprit de collection»). page 168 et ci-contre Boîte de coléoptères provenant de la collection d'André Breton, s.d. Collection particulière, photo Nelly Blaya/Musée de Cahors Henri-Martin.

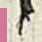

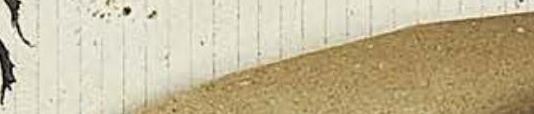

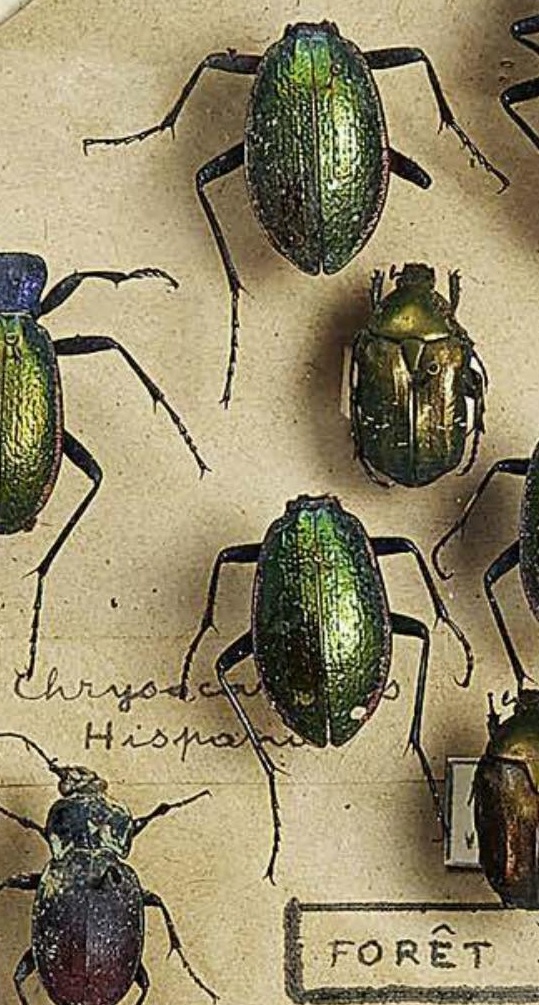




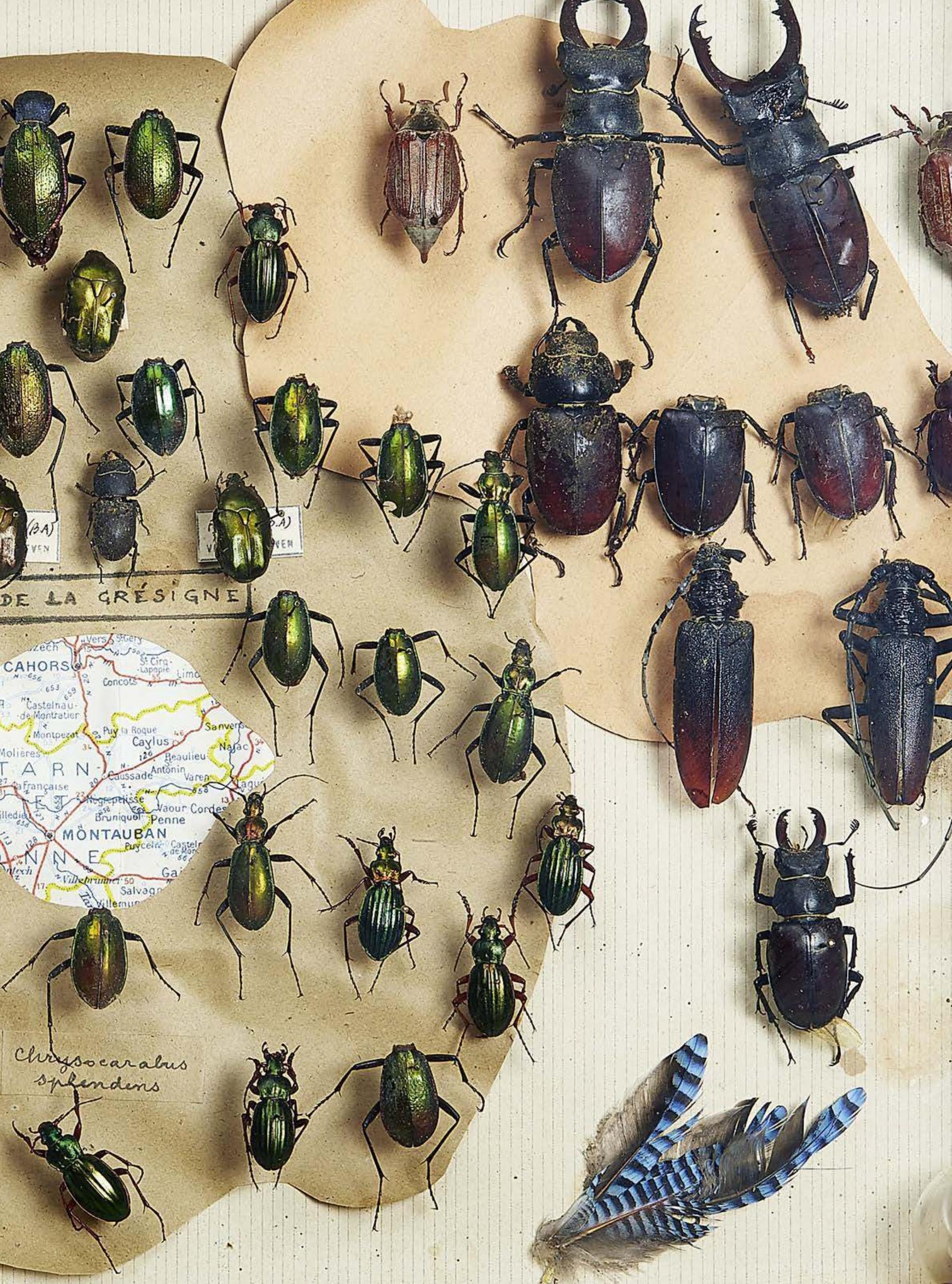

